\title{
A rotina ocupacional de pacientes implantados com cardiodesfibriladores ${ }^{1}$
}

\author{
Juliana Mannini, Janaína Santos Nascimento, Miryam Bonadiu Pelosi \\ Universidade Federal do Rio de Janeiro - UFRJ, Rio de Janeiro, RJ, Brasil
}

\begin{abstract}
Resumo: O cardiodesfibrilador implantável (CDI) se tornou referência quanto ao padrão de cuidado para pacientes com arritmias ventriculares potencialmente letais. O CDI trata os sintomas da doença sem qualquer impacto sobre a causa dela, porém a descarga elétrica advinda do mecanismo de funcionamento do dispositivo tem se mostrado fonte de muitos estudos referentes à qualidade de vida dessa população. A literatura aponta para limitações dos pacientes implantados com o cardiodesfribilador quanto à realização de atividades como trabalhar e dirigir, mas pouco se discute se outras áreas do desempenho ocupacional também estão limitadas. A pesquisa realizada foi prospectiva, de caráter exploratório, com o objetivo de conhecer a rotina de pacientes implantados com cardiodesfibriladores, com delineamento de estudo de caso e natureza quantitativa. Participaram da pesquisa 15 sujeitos, implantados com CDI e acompanhados ambulatorialmente pelo setor de arritmia do HUCFF. Os instrumentos de coleta de dados incluíram entrevista inicial, o inventário SF36, a Lista de Identificação dos Papéis Ocupacionais e uma questão aberta. Os resultados mostraram interferências na rotina ocupacional e desempenho dos papéis ocupacionais avaliados, assim como nos domínios de saúde mental e vitalidade, com maior enfoque nas influências advindas da patologia do que do próprio mecanismo do dispositivo.
\end{abstract}

Palavras-chave: Terapia Ocupacional, Cardiologia, Rotina.

\section{The occupational routine of patients implanted with cardioverter-defibrillator}

\begin{abstract}
Implantable cardioverter-defibrillators (ICD) have become a reference as to the standard of care for patients with potentially lethal ventricular arrhythmias. The ICD treats the symptoms of the disease with no impact on its cause; however, the electrical discharge originating from the operating mechanism of this device has been the source of many studies addressing the quality of life of this population. The specific literature points to limitations of patients implanted with cardioverter-defibrillators regarding activities such as working and driving, but little has been discussed whether other areas of occupational performance are also limited. The purpose of this prospective exploratory research, which uses a case study design of quantitative nature, is to know the routine of patients implanted with cardioverter-defibrillator. Fifteen subjects with ICD participated in the study; they were outpatients in the arrhythmia sector of the 'Clementino Fraga Filho' University Hospital - HUCFF. The instruments for data collection included an initial interview, SF36 inventory, Role Checklist, and an open question. The results showed interference in the routine occupational performance and occupational roles evaluated, as well as in the fields of mental health and vitality, with greater focus on influences arising from the pathology than the mechanism of the device.
\end{abstract}

Keywords: Occupational Therapy, Cardiology, Routine.

Autor para correspondência: Miryam Bonadiu Pelosi, Coordenação do Curso de Terapia Ocupacional, Prédio do CCS, Bloco K, Sala k49, Gabinete da Faculdade de Medicina da UFRJ, Rua Prof. Rodolpho Paulo Rocco, s/n, Cidade Universitária, Ilha do Fundão, Rio de Janeiro, RJ, Brasil, e-mail: miryam.pelosi@gmail.com

Recebido em Dez. 02, 2013; Revisão em Set. 08, 2014; Aceito em Out. 09, 2014. 


\section{Introdução}

\subsection{Cardiodesfibrilador implantável}

Michel Mirowski concebeu o primeiro Cardiodesfibrilador Implantável (CDI) após a morte de seu mentor, Harry Heller, que sucumbiu após um período de recorrentes taquiarritmias ventriculares. Ele imaginou um dispositivo cardíaco implantável que poderia tanto monitorar o ritmo cardíaco quanto acionar uma desfibrilaçáo ventricular apropriada. Os primeiros estudos com o dispositivo foram realizados em animais e levaram a uma tentativa humana bem-sucedida, sendo aprovado comercialmente nos EUA em 1985 (RAJ; SHELDON, 2001).

Segundo as Diretrizes da Sociedade Brasileira de Arritmias Cardíacas (Sobrac), o implante de cardiodesfibrilador é recomendado para sobreviventes de Infarto Agudo do Miocárdio há aproximadamente 40 dias, ou que apresentem cardiopatia isquêmica crônica, associada a tratamento farmacológico, sem isquemia miocárdica, passível de tratamento por revascularização cirúrgica ou percutânea e com expectativa de vida aproximada de um ano (LIMA; SILVA; MARTINELLI FILHO, 2009).

Desde a sua aprovação, em 1985, o cardiodesfibrilador implantável (CDI) se tornou referência quanto ao padrão de cuidado para pacientes com arritmias ventriculares potencialmente letais. O implante de cardiodesfibrilador tem se mostrado como uma ferramenta terapêutica importante para prevenir a morte súbita arrítmica em pacientes que apresentam doenças cardíacas orgânicas, com má função ventricular esquerda e/ou insuficiência cardíaca, mostrando-se um benefício profilático na mortalidade total dos pacientes que tenham alto risco de morte súbita (BILGE et al., 2006).

O aumento da popularidade de CDI decorre principalmente de sua segurança e tolerabilidade em comparação com os medicamentos comumente usados. As indicaçóes de CDI ampliaram o número de implantes e maior atençáo tem sido dirigida para sequelas da implantação. Embora escassos, os estudos de qualidade de vida em pacientes com CDI relatam consistentemente variados distúrbios psiquiátricos, como depressão e ansiedade (SOLA; BOSTWICK, 2005).

Os estudos de Sola e Bostwick mencionados abordam impactos negativos na qualidade de vida dos implantados com CDI. O aspecto mais relevante é a descarga elétrica esporádica do dispositivo, que é verificada como uma das maiores causas da redução da qualidade de vida desses pacientes (SOLA; BOSTWICK, 2005).

O estilo de vida desses pacientes se torna ainda mais comprometido devido ao funcionamento do dispositivo, que trabalha como uma espécie de "salva-vidas" no tratamento de pacientes com risco de vida (BILGE et al., 2006). Embora a maioria dos receptores de CDI apresentem níveis elevados de angústia imediatamente antes ou após a implantaçáo, essa tende a ser resolvida ao longo do tempo. Em estudo realizado para avaliar a qualidade de vida e bem-estar psicológico dos pacientes que tiveram a experiência de descargas elétricas de CDI nos primeiros 12 meses após o implante, constatou-se que os pacientes que sofreram descargas elétricas do CDI se adaptaram bem a viver com o dispositivo, mas não demonstraram maior ansiedade do que os destinatários de CDI que não receberam essas descargas elétricas. $\mathrm{A}$ expectativa de receber outra descarga elétrica, depois de experimentar uma, é menos estressante do que a da experiência da primeira descarga elétrica (KAMPHUIS et al., 2003).

O CDI trata os sintomas da doença, como arritmias ventriculares e morte súbita, sem qualquer impacto sobre a causa da doença. Esses sintomas, apesar de incomuns, apresentam características como imprevisibilidade, rapidez de início e potencial para a incapacitação rápida. Essas características conduzem a questôes éticas e legais sobre a importância da autorização para conduzir ou retornar ao trabalho após o implante do CDI (ANDERSON; CAMM, 1994).

Diante disso, o desenvolvimento do CDI aborda novos questionamentos, pois ao contrário de outras terapias existentes, não atua na prevenção de episódios arrítmicos, sendo sua função a de atuar diretamente na regulação rítmica cardíaca, no momento da arritmia.

O trabalho e o deslocamento através da condução de automóveis são atividades muito importantes para as tarefas cotidianas da maior parte das pessoas, contudo algumas doenças ou tratamentos médicos podem restringir essas atividades, apresentando um efeito adverso sobre a qualidade de vida das pessoas e implicaçôes econômicas para a sociedade como um todo. Essas restriçôes são impostas quando há um alto risco de lesão ou morte na realização dessas atividades.

Pessoas que são propensas à incapacitação súbita representam risco à sociedade, pois a maior parte está habilitada a dirigir e de maneira imprevisível 
podem causar acidentes graves. Pacientes com epilepsia fazem parte desse grupo e vários países têm regulamentos que regem a condução de veículos por pessoas epilépticas, por outro lado, o problema de pacientes com síncope causada por arritmias não recebe a mesma atenção. Em muitos países, os pacientes são proibidos de trabalhar após um episódio de arritmia por questóes de segurança pública; contudo, a posição no que diz respeito à condução de veículos é mais variável (ANDERSON; CAMM, 1994).

Estudos realizados no Hospital St. George, em 1992, constataram que dos 48 primeiros pacientes que receberam o implante de cardiodesfibrilador, $87 \%$ tiveram sua carteira de motorista detida, apesar de todos os participantes serem condutores ativos na época em que realizaram o implante do CDI. Dos $91 \%$ dos pacientes com menos de 65 anos de idade, 66\% estavam trabalhando até o implante do CDI, mas, após o implante, apenas $56 \%$ retornaram ao trabalho, enquanto $40 \%$ apresentaram restriçóes de circulação (ANDERSON; CAMM, 1994).

Assim, pode-se dizer que pacientes com CDI podem ter um impacto significativo na sua rotina diária, ocasionado pelas limitaçôes para condução de veículos e para o trabalho.

O aparelho cardiovascular é responsável pela distribuiçáo de oxigênio e nutrientes para todos os órgáos do corpo, sendo que qualquer ameaça ou distúrbio no funcionamento desse órgáo pode ocasionar impacto em sua eficiência, apresentando diferentes manifestaçóes, como fadiga muscular, dispneia, angina no peito, que contribuem para dificultar a execução do funcionamento ocupacional (CORDEIRO, 2007).

A atuação do terapeuta ocupacional na área da cardiologia, independentemente do diagnóstico, envolve uma avaliaçáo sobre os parâmetros clínicos que podem interferir na morbidade e na mortalidade desses pacientes, tais como as sequelas já instaladas no sistema cardiovascular, prognóstico do paciente, frequência cardíaca máxima e pressão arterial mínima, presença de quadros de depressão e ansiedade, entre outros. É fundamental um planejamento e intervençáo adequados de acordo com cada paciente, considerando os aspectos orgânicos, psicológicos e sociofamiliares dele, juntamente com a equipe de saúde que o atende, além de também considerar a estrutura institucional de tratamento existente, os recursos disponíveis e necessários, e a fase de tratamento em que o paciente se encontra (CORDEIRO, 2007).
No trabalho com pacientes com patologias cardíacas, o terapeuta ocupacional tem como principal objetivo a independência, a autonomia, a satisfação nas atividades e a participação social (CORDEIRO, 2007). De modo geral, o tratamento do terapeuta ocupacional com o paciente cardiopata busca conhecer, através de uma avaliação, questôes referentes ao estilo de vida e resposta cardiovascular a exercícios. Uma das abordagens realizadas pelo profissional durante a internaçáo do paciente cardiopata é a estruturação e organização da rotina do paciente, a partir de uma tabela de planejamentos de atividades (reabilitação, nutrição, intervençóes, educaçáo, tratamentos, consideraçóes espirituais e psicossociais, planejamento da alta), assegurando dessa forma um nível padronizado de cuidados. $\mathrm{O}$ planejamento para a alta visa orientar o paciente sobre seu nível funcional físico, assim como um programa domiciliar detalhado e individualizado sobre atividades e exercícios, simplificação e ritmo de trabalho, precauçóes com a temperatura, atividade social, sexualidade, sinais e sintomas de intolerância ao exercício e/ou discussão sobre fatores de risco (HUNTLEY, 2005).

A literatura aponta para limitaçóes dos pacientes implantados com o cardiodesfribilador quanto à realizaçáo de atividades como trabalhar e dirigir, mas pouco discute se outras áreas do desempenho ocupacional também estão limitadas. A hipótese deste estudo foi a de que o implante do cardiodesfibrilador influencia negativamente na rotina ocupacional dos pacientes e em outras áreas do desempenho como as atividades de vida diária, o estudo, o lazer e as interaçóes sociais.

O Hospital Universitário Clementino Fraga Filho (HUCFF) é referência quanto ao implante de cardiodesfibriladores no estado do Rio de Janeiro. Quanto ao acompanhamento clínico de pacientes portadores do CDI no HUCFF, sabe-se que depois do implante do aparelho e a alta hospitalar, que acontece entre 24 a 48 horas após a cirurgia, são realizadas avaliaçóes periódicas desses pacientes, pela equipe médica, a 7, 15 e 30 dias da intervenção. Depois desse primeiro mês, os pacientes são avaliados a cada três meses quanto aos acionamentos do CDI, quando também é acessada a mortalidade global e arrítmica dos portadores do aparelho (FONSECA et al., 2007)

Em levantamento preliminar acessando o banco de dados no setor de arritmia do HUCFF, tem-se que foram realizados 150 procedimentos de implantes de CDI de janeiro de 2006 a dezembro de 2012 , sendo que $41,3 \%$ referiam-se à troca do 
dispositivo. Dessa forma, faz-se necessário um acompanhamento do paciente após a cirurgia, a fim de verificar possíveis impactos que o implante do cardiodesfibrilador possa trazer à rotina desses pacientes.

\section{Objetivo}

Avaliar quais as áreas do desempenho ocupacional se apresentam mais impactadas na rotina ocupacional dos sujeitos participantes antes e depois do implante do cardiodesfibrilador.

\section{Metodologia}

Foi realizada uma pesquisa prospectiva, de caráter exploratório com delineamento de estudo de caso e natureza quantitativa.

Os instrumentos de coleta de dados incluíram entrevista inicial, uma questão aberta, o inventário SF36 e a Lista de Identificação de Papéis Ocupacionais.

As questóes fechadas referentes aos instrumentos foram analisadas de forma descritiva por meio de frequência absoluta. A mesma análise foi realizada para a questáo aberta, após a organização das respostas em categorias.

\subsection{Sujeitos}

Participaram da pesquisa 15 adultos com mais de 18 anos, implantados com o CDI há mais de três meses e que eram acompanhados ambulatorialmente pelo setor de arritmia do HUCFF.

Foram incluídos os pacientes que apresentavam as características descritas acima e que assinaram o Termo de Consentimento Livre e Esclarecido (TCLE). Foram excluídos da pesquisa os sujeitos menores de 18 anos, com implante há menos de três meses, que apresentavam déficit cognitivo, que não estavam em acompanhamento ambulatorial no HUCCF ou que não aceitaram participar da pesquisa.

\subsection{Local}

A pesquisa foi desenvolvida por uma terapeuta ocupacional integrante da Residência Multiprofissional do Hospital Universitário Clementino Fraga Filho em janeiro e fevereiro de 2013, após a autorização, por meio do número 109-12, pelo Comitê de Ética e Pesquisa da UFRJ.

\subsection{Procedimentos}

Inicialmente foi realizada uma entrevista com os sujeitos participantes da pesquisa, depois da assinatura de um Termo de Consentimento Livre e Esclarecido, buscando as informaçóes nome, idade, estado civil, profissão, diagnóstico clínico e período em que realizou o implante do cardiodesfibrilador, com o objetivo de traçar o perfil da população avaliada. A avaliação foi realizada no ambulatório de arritmia do $2^{\circ}$ andar do HUCFF, às quartasfeiras, no período das 8 às 11 h, em sala individual, apenas com a presença do entrevistado. O tempo estimado para cada entrevista foi de 20 a 30 minutos e a cada semana eram entrevistados dois pacientes, sendo que a quantidade de entrevistados era relativa ao número de pacientes implantados com o $\mathrm{CDI}$ que realizavam consulta médica no dia. Os médicos responsáveis pelo setor os encaminhavam para a pesquisa quando finalizada a consulta.

Após a entrevista inicial foi aplicado o questionário de avaliaçáo SF36 (Short-Form Health Survey 36), que se refere aos domínios de saúde mental e vitalidade.

Em seguida foi realizada a aplicaçáo, por meio de entrevista, do questionário denominado Lista de Identificação de Papéis Ocupacionais, traduzido e adaptado culturalmente para a língua brasileira por Cordeiro (2005). Os períodos referentes à avaliação foram estabelecidos como passado (anterior ao implante), presente (após o implante, atual) e futuro (perspectivas futuras relacionadas a um período de aproximadamente cinco anos).

Para a análise a respeito do desempenho ocupacional dos participantes, tendo como referência a fase anterior ao implante e posterior a ele, foi aplicada uma questáo aberta, a qual teve as respostas transcritas pelos participantes ou examinadora, quando eles se apresentaram com dificuldades para a escrita ou não demonstravam disposição para executá-la.

\subsection{Instrumentos}

- O SF36 foi criado a partir de uma revisão de instrumentos relacionados à qualidade de vida já existentes na literatura nos últimos 20 anos. É um inventário que avalia oito domínios distintos: capacidade funcional, aspectos físicos, aspectos emocionais, dor, estado geral de saúde, vitalidade, aspectos sociais e saúde mental, os quais se encontram disponíveis nas 10 questóes fechadas do inventário. Os 
itens avaliados apresentam um resultado para cada questão, que após a aplicação são transformados em valor numérico, numa escala de 0 a 100 , em que 0 é considerado o pior estado e 100, o melhor. Os domínios avaliados para essa pesquisa foram somente os da saúde mental e vitalidade, incluídos apenas na questão 9, devido à relevância sobre a relação entre o estado emocional e a qualidade de vida, para identificar o quanto tais domínios foram influenciados após o implante do CDI (TEIXEIRA; FONSECA; MAXIMO, 2002).

- A Lista de Identificação de Papéis Ocupacionais foi baseada em conceitos descritos no Modelo de Ocupação Humana. A aplicação da avaliação é realizada a partir de duas etapas, a primeira consiste na definição do cliente de sua participação ou não em papéis ocupacionais (estudante, trabalhador, voluntário, cuidador, serviço doméstico, amigo, membro de família, religioso, passatempo/amador, participante de organizaçóes e outros) no passado, presente e futuro. A segunda etapa consiste na atribuiçáo do grau de importância pelo cliente a cada papel descrito (desempenhado e não desempenhado), havendo ou não variação em relação à importância atribuída. $\mathrm{O}$ protocolo disponibiliza questóes fechadas, pode ser utilizado com adolescentes, adultos e idosos, é autoplicável e sua administração exige um tempo de duração curto (CHAVES et al., 2010).

- A questão aberta sobre a rotina ocupacional dos pacientes abordou aspectos referentes às possíveis atividades ocupacionais que estariam prejudicadas após o implante do cardiodesfibrilador e constava a seguinte pergunta: "Em relação às atividades que você costumava desempenhar do início ao final do seu dia, houve alteração em alguma dessas atividades após o implante do cardiodesfibrilador? Em caso de resposta afirmativa, em quais atividades?"

\section{Resultados}

\subsection{Entrevista}

Através da entrevista inicial realizada com os 15 participantes, dos quais oito eram do sexo masculino e sete do sexo feminino, com faixas etárias de 24 a 87 anos, foram coletadas as informaçóes a seguir;

Os participantes entrevistados apresentavam diagnósticos variados como cardiopatia chagásia com disfunção leve ou grave do ventrículo esquerdo, displasia e disfunção do ventrículo direito com envolvimento do ventrículo esquerdo, ventrículo esquerdo náo compactado com disfunção grave, cardiopatia isquêmica e cardiopatia dilatada. Dos 15 sujeitos entrevistados, nove eram casados, quatro, solteiros, um, divorciado e um, viúvo. Com relação à atividade profissional, os participantes citaram como atividades profissionais mais recentemente desempenhadas: copeira $(\mathrm{N}=1)$, dona de casa $(\mathrm{N}=4)$, costureira $(\mathrm{N}=1)$, diarista $(\mathrm{N}=1)$, comerciante $(\mathrm{N}=1)$, padeiro $(\mathrm{N}=1)$, vigia $(\mathrm{N}=1)$, auxiliar administrativo $(\mathrm{N}=1)$, operário de obra $(\mathrm{N}=4)$, sendo que 11 dos entrevistados se encontravam inativos e quatro atuavam na profissão. Dos inativos, 10 estavam aposentados por invalidez. Com relação ao implante de CDI, seis dos entrevistados haviam realizado a troca do aparelho, enquanto nove haviam implantado o aparelho somente uma vez, sendo que o período em que se encontravam implantados variou de 3 meses a 10 anos.

\subsection{SF 36}

Os resultados relacionados aos domínios de saúde mental e vitalidade do SF36 foram calculados conforme a ponderação dos dados referentes ao score correspondente às alternativas de cada domínio. Foi realizada a chamada raw scale, em que valores efetuados variaram de 0 a 100 , em que 0 correspondeu ao pior score e 100 ao melhor para cada domínio. É chamado de raw scale porque o valor final não apresenta nenhuma unidade de medida.

\section{- Domínio de Saúde Mental}

Os valores referentes a esse domínio, apresentados na Figura 1, variaram de 8 a 80 . Sendo que 10 dos entrevistados apresentaram valores abaixo da média de 50 e cinco dos entrevistados apresentaram valores acima.

\section{- Domínio da Vitalidade}

Os valores referentes a esse domínio variaram de 5 a 100 e nove dos entrevistados apresentaram valores abaixo da média de 50 ; seis dos entrevistados apresentaram valores acima da média, conforme a Figura 2. 


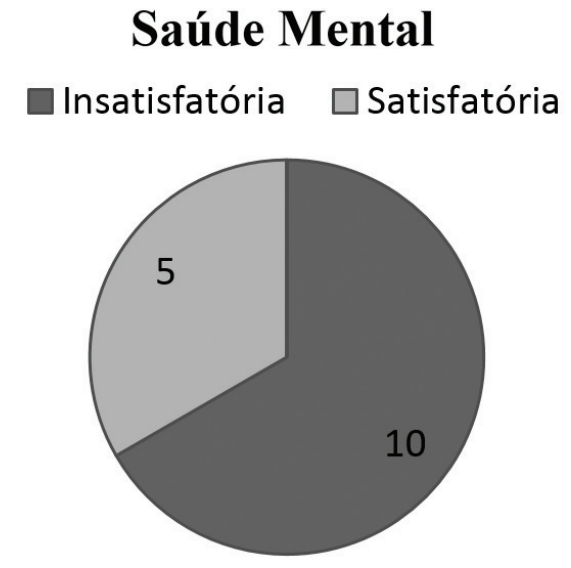

Figura 1. Domínio de Saúde Mental.

\section{Vitalidade}

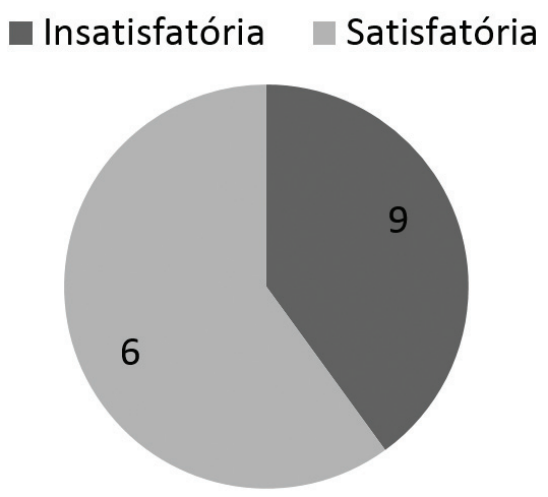

Figura 2. Domínio Vitalidade.

\subsection{Lista de papéis ocupacionais}

Os 10 papéis ocupacionais listados foram observados e anotados segundo os critérios descritos na metodologia, tendo como referência os papéis ocupacionais anteriores ao implante (passado), os papéis ocupacionais exercidos após o implante, ou seja, atuais (presente), e os papéis ocupacionais almejados futuramente. Nesses últimos, por um período de aproximadamente 5 anos, diante da nova perspectiva de vida que as possibilidades e impossibilidades do CDI pode ofertar (futuro).

A incumbência percebida dos papéis ocupacionais está apresentada na Tabela 1 .
Com relação à importância designada para cada papel, os resultados são apresentados na Tabela 2 .

Quanto aos resultados da questão aberta, tem-se que com relaçáo às respostas dos participantes, todos referiram que apresentavam modificaçóes quanto à sua rotina ocupacional após o implante do cardiodesfibrilador, sendo que três dos entrevistados referiram que houve alteraçóes quanto às atividades sexuais, e sete dos entrevistados, quanto às atividades domiciliares. Os dados estão apresentados na Figura 3.

\section{Discussão}

Considerando-se os dados a respeito do papel estudante, a maior parte dos entrevistados $(\mathrm{N}=12)$ já havia realizado esse papel antes do implante. Dos três restantes, apenas um não havia estudado e também não designava nenhuma importância a esse papel ocupacional, um deles já havia estudado e tinha como expectativa retornar e apenas um participante continuava a estudar após o implante do CDI. O implante do CDI não se mostrou limitador para o desempenho desse papel, mas mais de $90 \%$ dos participantes náo demonstraram interesse em retomar esse papel ocupacional, sem que outras correlaçốes a respeito do tema fossem aprofundadas.

Em contrapartida, o papel ocupacional trabalhador apresentou-se como uma atividade prejudicada pelo implante do CDI. Dos entrevistados, 11 não estavam mais trabalhando, sendo que, desse grupo, 10 estavam aposentados por invalidez. As quatro pessoas do grupo pesquisado que ainda trabalhavam exerciam funções como copeira $(\mathrm{N}=1)$, operário de obra $(\mathrm{N}=1)$ e comerciante $(\mathrm{N}=2)$, com idade, respectivamente, de 56 anos, 51 anos, 65 anos e 70 anos. Os entrevistados que trabalhavam como comerciantes e já estavam aposentados, apenas modificaram suas funçóes anteriores a fim de continuar a desempenhar o papel ocupacional com menor gasto energético. A entrevistada que trabalhava como copeira relatou que essa função não lhe era prejudicial à saúde e não se mostrava limitada pelo $\mathrm{CDI}$ ou doença. O paciente que trabalhava em obra não estava aposentado $\mathrm{e}$, devido a sua condição socioeconômica, continuava a trabalhar na mesma função que realizava anteriormente, obtendo auxílio dos colegas de serviço para a realizaçấo de atividades que apresentavam maior gasto energético.

Em estudo realizado por Dantas, Aguillar e Barbeira (2001) sobre o retorno ao trabalho após 
Tabela 1. Distribuição dos papéis ocupacionais - incumbência percebida $(\mathrm{n}=15)$.

\begin{tabular}{lcccccccc}
\hline \multicolumn{1}{c}{ Variáveis } & \multicolumn{7}{c}{ Incumbência percebida } \\
\hline \multicolumn{1}{c}{ Papéis ocupacionais } & PA & PAPRE & PAFU & PRE & PREFU & FU & PAPREFU & NA \\
\hline Estudante & 12 & 0 & 1 & 1 & 0 & 0 & 0 & 1 \\
Trabalhador & 9 & 1 & 0 & 1 & 0 & 1 & 2 & 1 \\
Voluntário & 2 & 0 & 1 & 0 & 0 & 3 & 0 & 9 \\
Cuidador & 1 & 2 & 0 & 9 & 1 & 0 & 0 & 2 \\
Serviço doméstico & 4 & 0 & 0 & 9 & 0 & 0 & 1 & 1 \\
Amigo & 5 & 1 & 0 & 4 & 0 & 0 & 0 & 5 \\
Membro da família & 0 & 0 & 0 & 10 & 3 & 0 & 1 & 1 \\
Religioso & 1 & 0 & 0 & 9 & 1 & 1 & 0 & 3 \\
Passatempo/ Amador & 1 & 0 & 2 & 4 & 0 & 1 & 0 & 7 \\
Participante de & 1 & 0 & 0 & 0 & 0 & 1 & 0 & 13 \\
organizações & & & & & & & & \\
Outros & 0 & 0 & 0 & 0 & 0 & 0 & 0 & 15 \\
\hline
\end{tabular}

PA - papel ocupacional realizado somente no passado; PAPRE - papel ocupacional desempenhado no passado e no presente, sem pretensões de continuar a desempenhá-lo no futuro; PAFU - papel ocupacional desempenhado apenas no passado, com pretensão de voltar a desempenhá-lo no futuro; PRE - papel ocupacional realizado somente no presente; PREFU - papel ocupacional desempenhado apenas no presente, com pretensão de desempenhá-lo no futuro; FU - pretensão de realizar o papel ocupacional apenas no futuro; PAPREFU - papel desempenhado no passado e no presente, com pretensões de continuar a desempenhá-lo no futuro); e NA - papel nunca desempenhado no passado, no presente e sem pretensões de desempenhá-lo no futuro

Tabela 2. Distribuição dos papéis ocupacionais - importância designada ( $\mathrm{n}=15)$.

\begin{tabular}{lccc}
\hline \multicolumn{1}{c}{ Variáveis } & Importância designada & \\
\hline Estudante & Nenhuma & Alguma & Muita \\
Trabalhador & 1 & 4 & 10 \\
Voluntário & 0 & 1 & 14 \\
Cuidador & 0 & 2 & 13 \\
Serviço doméstico & 1 & 0 & 14 \\
Amigo & 0 & 6 & 9 \\
Membro da família & 2 & 3 & 10 \\
Religioso & 0 & 0 & 15 \\
Passatempo/Amador & 0 & 1 & 14 \\
Participante em organizações & 0 & 1 & 14 \\
Outros & 12 & 1 & 2 \\
\hline
\end{tabular}

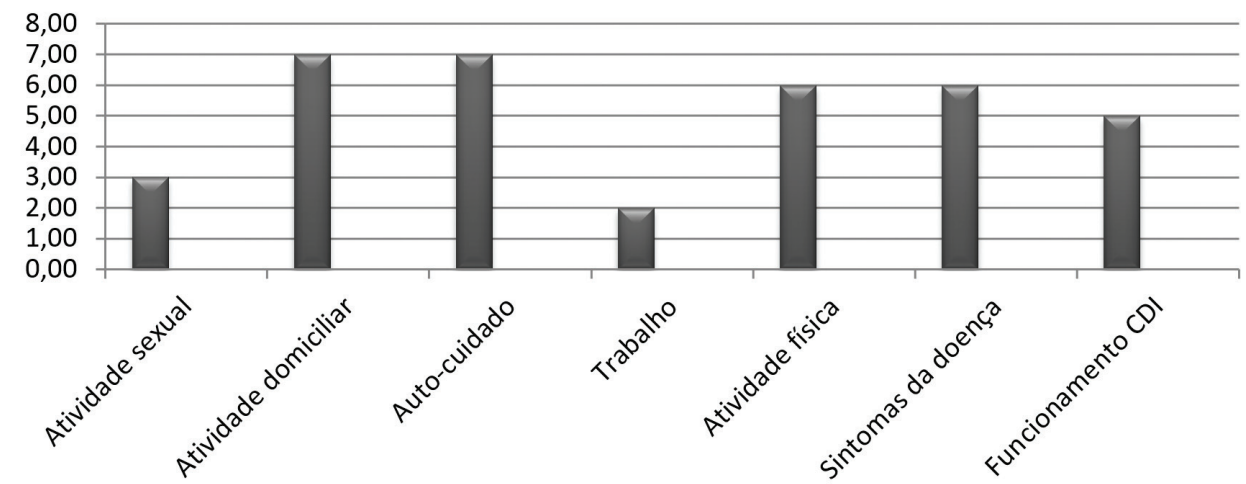

Figura 3. Resultados da questão aberta. 
eventos cardíacos, constatou-se que algumas variáveis devem ser consideradas, tais como as demográficas (idade, sexo, situação socioeconômica, estado civil e tipo de profissão), psicossociais (depressão, apoio social, percepçáo do paciente com relaçáo à sua capacidade de trabalho e sua autoeficiência); as relacionadas ao contexto do trabalho (satisfação com o emprego, demandas físicas e tensóes da profissão); e também aos fatores clínicos (gravidade da doença, dor, capacidade funcional e influências do tratamento, como restriçôes médicas e efeitos de medicamentos), verificando-se que as questóes relacionadas ao contexto do trabalho foram consideradas por uma minoria. O referido estudo também aponta que trabalhadores do sexo masculino, com profissões melhor remuneradas, mais intelectuais e com status mais reconhecido, retornam ao trabalho com maior frequência que trabalhadores braçais e pacientes do sexo feminino. Aqueles que também apresentam maior apoio social retornam ao trabalho mais rapidamente, assim como os fatores obrigaçóes familiares e compromissos financeiros podem também estimular o retorno mais precocemente à ocupação. Dessa forma, assim como no estudo citado, a continuidade ou o retorno ao trabalho foi realizado pela minoria dos sujeitos entrevistados, que se enquadraram em atividades que exigiam maior conservação de energia e maior apoio social dos colegas de trabalho.

O papel de cuidador foi relatado como desempenhado, após o implante do CDI, por 12 dos entrevistados.

Para a grande maioria do grupo entrevistado, 14 dos 15 participantes, o papel de membro da família foi muito importante e possível de ser desempenhado após o implante do CDI.

Os dados do papel ocupacional religioso mostraram uma aproximação significativa ao desempenho do papel membro da família após o implante do CDI, já que 10 entrevistados do grupo não haviam desempenhado esse papel no passado e passaram a exercê-lo após o implante do CDI, como um papel atual. Apenas um participante assinalou o desejo de continuar a desempenhar esse papel no futuro. Para somar a esse número, um dos participantes, que ainda não desempenhava o papel religioso, pensava em fazê-lo no futuro. A importância designada para o papel foi muito importante para 14 dos entrevistados.

Os dados sobre o desempenho nos serviços domésticos apontam que para 10 dos entrevistados o implante do CDI náo afetou suas possibilidades de realização de trabalhos domésticos. A maior parte
$(\mathrm{N}=9)$ dos que responderam que realizavam o papel no presente passou a realizá-lo apenas depois que implantaram o CDI. Para quatro entrevistados, o papel foi desempenhado apenas no passado.

A lista de papéis ocupacionais mostrou que o papel relacionado ao desempenho no serviço doméstico não foi prejudicado após o implante do $\mathrm{CDI}$, no entanto, a qualidade do desempenho desses papéis foi citada como limitada após o implante do CDI na questão discursiva respondida pelos participantes. As Atividades Instrumentais da Vida Diária, como a prática de exercícios físicos e a execuçáo de serviços domésticos foram citadas por seis e sete participantes, respectivamente, na questão aberta sobre atividades realizadas anteriormente ao implante do CDI que se tornaram impossíveis de realizar após o implante.

De acordo com estudo realizado por Levy et al. (1999, p. 2):

Em se tratando do metabolismo aeróbico, quanto mais eficiente for o sistema cardiovascular em oferecer oxigênio e distribuir o fluxo sanguíneo aos tecidos, melhor será a capacidade funcional desse sistema (maior eficiência na extração e transporte de oxigênio pelos tecidos em atividades metabólicas acima dos níveis de repouso). Durante a execução de atividade/ tarefa, aumenta o consumo de oxigênio pelos músculos esqueléticos na proporçáo da intensidade dessa atividade.

Dessa forma, tem-se que mesmo as atividades domésticas podem ocasionar dificuldades após o implante do CDI, em especial quando realizadas simultaneamente, devido ao gasto energético de cada atividade, que pode ser estimado com o Equivalente Metabólico da Tarefa (MET).

Os pacientes pontuaram dificuldades para: varrer a casa (3,0 MET); lavar banheiro (3,5 MET); estender tapete e roupas (3,0 MET); fazer comida (2,5 MET); passar roupa (3,5 MET); ou realizar exercícios como subir e descer escadas (5,0 MET); carregar peso (7,0 MET); andar de bicicleta (4,0 MET); abaixar-se e levantar-se $(3,0)$; e correr (4,5 MET). Ressalta-se que 1 MET corresponde a $3,5 \mathrm{ml} / \mathrm{kg} / \mathrm{min}$ de consumo de oxigênio (BARTHOLOMEW et al., 2011).

Exercícios físicos podem gerar demasiada insegurança nos sujeitos portadores de doenças cardíacas. Os pacientes ficam temerosos quanto ao aumento do grau da força ao nível do coração, preferindo náo se expor a atividades rotineiras que possam requerer um maior gasto de oxigênio e, 
dessa forma, sobrecarregar o sistema cardiovascular acometido pela patologia (ALBANESI-FILHO, 2000).

O exercício é reconhecido como um grande contributivo na gênese de morte súbita em alguns subgrupos de indivíduos. Tem-se que algumas condutas médicas adotam a não prática dos exercícios como orientaçâo mais aconselhável no caso das doenças cardíacas. Entretanto, se o exercício físico pode aumentar transitoriamente o risco relativo de eventos, o risco absoluto permanece muito baixo e a probabilidade de que ocorram tais eventos é muito pequena (BRONZATTO; SILVA; STEIN, 2001).

O papel ocupacional de voluntário teve importância significativa $(\mathrm{N}=13)$ para os entrevistados, porém somente três deles pensavam desempenhá-lo no futuro. Nenhum dos entrevistados realizava o papel no presente após o implante do CDI. Para a maioria $(\mathrm{N}=9)$, esse papel não tinha sido realizado e essa maioria nem cojitava realizá-lo no futuro.

O papel ocupacional de participante de organizaçôes também não foi desempenhado por nenhum dos entrevistados após o implante do CDI, e para 13 entrevistados do grupo, nunca foi considerado no passado e nem como atividade futura.

O papel de passatempo/amador foi relatado como desempenhado no presente por apenas quatro pessoas do grupo entrevistado, e por três deles com pretensóes de realizaçáo no futuro. Para a maioria $(\mathrm{N}=7)$, ele não foi considerado em nenhuma etapa da vida. Apenas para um participante esse papel ocupacional foi desempenhado somente no passado, e interrompido.

O papel de amigo foi desempenhado após o implante do CDI por cinco dos entrevistados, sendo que outros cinco relataram já o terem realizado somente no passado. Para cinco dos entrevistados, ele não havia sido cogitado em nenhuma etapa da vida. O papel náo demonstrou receber interferências do implante do CDI.

Os papéis ocupacionais de voluntário, passatempo/amador, participante em organizaçóes e amigo foram citados por mais de $50 \%$ dos participantes como nunca desempenhados no passado nem no presente, citando-se adicionalmente que também não tinham pretensóes de desempenhá-los no futuro. Dessa maneira, esses papéis ocupacionais não se mostraram relevantes para a pesquisa, já que não apresentaram dados relacionados ao implante do CDI mas sim às características individuais a respeito da importância no desempenho deles. Tais papéis não foram abordados na questão aberta.

O desempenho dos papéis ocupacionais se torna o principal foco de intervenção dos terapeutas ocupacionais para o retorno às funçóes que antes eram importantes componentes de autoimagem dos sujeitos assistidos, e que se tornam prejudicadas após o sofrimento de traumas ou doenças crônicas que geram alteraçóes corporais. É o terapeuta ocupacional o principal responsável pela promoção ou restauração adequada do desempenho nessas atividades, visando a maior independência e satisfação das necessidades e objetivos de sua clientela (CORDEIRO, 2005).

A importância designada a cada papel foi semelhante aos resultados obtidos na incumbência percebida, já que os papéis referidos que apresentaram maior número de participantes ativos antes do implante do CDI, ou mesmo após o implante, foram também os referidos pelos participantes como os de maior importância.

A maior parte do grupo estudado passou a desempenhar, após o implante do CDI, os papéis membro da família $(\mathrm{N}=14)$, cuidador $(\mathrm{N}=12)$, trabalhador doméstico $(\mathrm{N}=10)$ e religioso $(\mathrm{N}=10)$. Em menor quantidade, os papéis amigo $(\mathrm{N}=5)$, trabalhador $(\mathrm{N}=4)$, passatempo/amador $(\mathrm{N}=4) \mathrm{e}$ estudante $(\mathrm{N}=1)$. Os entrevistados náo incluíram, após o implante do $\mathrm{CDI}$, os papéis voluntário e participante de organizaçôes $(\mathrm{N}=0)$.

Com relaçáo ao desempenho anterior ao implante do CDI, os papéis que se mostraram mais importantes para o grupo foram os de estudante $(\mathrm{N}=12)$ e trabalhador $(\mathrm{N}=9)$. Com uma quantidade bem menor, serviço doméstico $(\mathrm{N}=4)$, amigo $(\mathrm{N}=4)$, voluntário $(\mathrm{N}=2)$, cuidador $(\mathrm{N}=1)$, religioso $(\mathrm{N}=1)$, passatempo/amador $(\mathrm{N}=1)$, participante em organizaçóes $(\mathrm{N}=1)$ e outros $(\mathrm{N}=1)$. Os entrevistados não incluíram o papel membro da família como papel realizado anteriormente ao implante do CDI.

A área de desempenho ocupacional das Atividades de Vida Diária relacionada às atividades sexuais e a de autocuidado foram mencionadas por três e seis participantes, respectivamente, como atividades que eram desempenhadas antes do implante e que passaram a não ser mais realizadas depois dele. Essa foi uma atividade que apresentou significativa importância no discurso dos indivíduos participantes da pesquisa. 
As atividades elencadas na questão discursiva estavam relacionadas às tarefas de autocuidado como:

andar (2,3 MET); conversar (3,0 MET) tomar banho (3,0 MET); lavar o rosto (2,0 MET); escovar os dentes (2,0 MET); fazer a unha (2,0 MET); e rezar (2,0 MET) (BARTHOLOMEW et al., 2011, p. 3-4).

Por serem atividades que demandam gasto energético, mesmo que mínimo, são também importantes de serem consideradas no processo do funcionamento cardiovascular dos pacientes implantados com cardiodesfibrilador, pois eles apresentam respostas diferenciadas do aparelho, devido à própria cardiopatia e ao funcionamento cardíaco característico de cada sujeito.

Muitos estudos têm abordado o assunto referente às atividades sexuais após eventos cardíacos. Uma quantidade significativa de pacientes não retorna às atividades sexuais após a ocorrência da doença. Alguns fatores associados às mudanças fisiológicas decorrentes do processo de envelhecimento, disfunçóes induzidas por medicamentos e mudanças vasculares relativas a fatores de risco como diabetes, hipertensão ou dislipidemias, adicionadas às questôes psicológicas da cardiopatia, são aspectos a serem considerados quanto à incapacidade dos pacientes realizarem atividades sexuais (DANTAS; AGUILAR; BARBEIRA, 2001).

O esclarecimento a respeito da retomada da atividade sexual desses pacientes é de extrema necessidade, já que eles acabam não retomando a vida sexual por acreditarem que ela é uma atividade de risco.

Informações de que o esforço físico, no início do ato sexual, equivale a atividades cotidianas como a de andar 200 a 300 metros no plano ou subir dois lances de escada são fundamentais (MACHADO; PELÁ, 2001). Outras orientaçôes dadas no início do tratamento aos pacientes e parceiros podem auxiliar. São elas: diminuir o esforço despendido no ato sexual; não realizar atividades sexuais após a refeição, após o consumo excessivo de álcool, em temperaturas extremas, ou períodos de fadiga pronunciada.

Dessa forma, a cronicidade da doença náo deve ser considerada um fator limitante para a relaçáo sexual, desde que orientada adequadamente (STEIN; HOHMANN, 2006).

Ainda em relação à questão discursiva, seis dos entrevistados mantiveram respostas referentes à modificação da rotina ocupacional devido aos sintomas decorrentes da patologia. Os fatores relacionados aos sintomas citados foram tonteira, desmaios, fadiga e cansaço, sintomas esses que podem ser controlados com o implante do CDI, já que o mesmo também atua no controle do ritmo cardíaco, prevenindo as sensaçôes de mal-estar típicas das alteraçôes na frequência cardíaca. Em relação às atividades que desencadeiam tais sintomas, essas devem ser orientadas e adequadas de acordo com a possibilidade de cada sujeito avaliado, conforme discutido anteriormente.

As respostas de cinco entrevistados referentes ao funcionamento do CDI na questão discursiva estiveram relacionadas à descarga elétrica proporcionada pelo aparelho, assim como ao sinal indolor que o mesmo produz inicialmente para o controle do ritmo cardíaco.

A resposta de um paciente a partir da recuperação da descarga elétrica do CDI deve ser considerada como um evento crítico, com implicações de prognóstico. A ansiedade relacionada com o disparo do CDI é o único aspecto mais perturbador do tratamento.

O nível de perigo não depende do número de descargas elétricas que o paciente recebe, mas pode ser baseado na interpretação catastrófica do paciente em relação a experiência (SOLA; BOSTWICK, 2005).

Arteaga-Fernández (2011) realizou um estudo no Instituto do Coração do Hospital das Clínicas de São Paulo sobre o efeito do CDI na sobrevida e as complicaçôes por ele determinadas em pacientes com cardiomiopatia hipertrófica que receberam o dispositivo para prevenção primária e secundária. Os resultados mostraram que o tratamento do CDI na prevenção da morte súbita é eficiente, porém ainda limitado pela elevada ocorrência de complicaçôes do sistema gerador cabo-eletrodo, o qual determina choques inapropriados, devendo ser indicado preferencialmente na prevenção secundária somente para casos selecionados na prevenção primária. O estudo também detectou que a maioria dos casos que apresentaram choques inapropriados estava relacionada a pacientes que realizaram a troca de CDI.

Estudo que avaliou as prováveis interferências eletromagnéticas nos pacientes implantados com cardiodesfibriladores ou com marcapassos cardíacos nas atividades de vida diária e nas de trabalho verificou que a reeducação dos pacientes, pela equipe de saúde, era um excelente encaminhamento para minimizar os problemas encontrados ante a grande exposição de sinais eletromagnéticos 
que permeiam a rotina diária desses indivíduos (FERNÁNDEZ-BANIZI et al., 2004).

De acordo com estudo feito em São Paulo sobre as situaçóes mais citadas pelos pacientes como responsáveis pela alteração do funcionamento do marcapasso, foram encontrados fatores como o stress, choque elétrico, pegar peso e uso do forno de microondas. Faz-se dessa forma necessária a orientação a respeito de um menor contato com alguns equipamentos eletrônicos e orientaçóes sobre técnicas de conservação de energia em atividades que confiram maior gasto energético dessa população (BRASIL; CRUZ, 2000).

O questionário de qualidade de vida SF36, nos domínios de vitalidade e saúde mental, mostrou mais da metade dos participantes apresentando aspectos referentes à vitalidade e saúde mental como insatisfatórios.

Os problemas psicológicos mais comuns, após o implante de CDI, são: ansiedade, depressão, sentimentos antecipatórios sobre o disparo do dispositivo e medo da morte. Também é muito comum sentir raiva e apresentar alteraçóes na imagem corporal (SOLA; BOSTWICK, 2005).

Poucos autores têm documentado a incidência específica do implante de CDI induzindo a ansiedade. No entanto, um número pequeno de estudos aborda o tema geral sobre qualidade de vida em pacientes com CDIs. Todos esses estudos compartilham a debilidade de confiança sobre as medidas qualitativas e que apresentam caráter mais retrospectivo. Apenas um estudo não encontrou diferenças de qualidade de vida entre os grupos com e sem disparos do dipositivo (DURU et al., 2001).

Embora a aceitação do CDI seja geralmente boa devido à possibilidade da morte por arritmia, receios quanto ao implante do dispositivo são comuns (EADS et al., 2000). A maioria dos estudos conclui que o implante do CDI é benéfico para a qualidade de vida dos pacientes em comparação com aqueles que náo o implantaram. No entanto, baixos níveis de suporte social, alto nível de ansiedade e número elevado de disparos do dispositivo estão associados à má qualidade de vida dessa população (SEARS JUNIOR; CONTI, 2006).

\section{Considerações finais}

Dessa forma, conclui-se que apesar do dispositivo se mostrar benéfico em relação à melhoria do funcionamento cardíaco, o mesmo se mostrou prejudicial no desempenho satisfatório dos papéis ocupacionais dos pacientes que realizaram o procedimento, já que muitos acabaram por diminuir suas atividades rotineiras após o implante do dispositivo.

No entanto, deve-se considerar que a patologia associada ao uso do CDI pode ser a maior limitadora das atividades ocupacionais dessa população, sendo indiferente ao mecanismo de ação do cardiodesfibrilador, já que a própria doença aumenta o impacto negativo durante a realização dessas atividades, em função dos sintomas que a ela estão associados.

Os aspectos relacionados à qualidade de vida, como a saúde mental e vitalidade, também se mostraram afetados após o implante. De modo geral, se mostraram vinculados à diminuição das atividades ocupacionais realizadas anteriormente ao implante e, possivelmente, à descoberta do diagnóstico.

Espera-se que os resultados produzidos neste estudo sejam disparadores de outras pesquisas que busquem estratégias de melhoria da qualidade de vida de pessoas com alteraçóes do funcionamento cardíaco e que se encontrem implantadas com o cardiodesfibrilador. Com o avanço dos estudos, os efeitos poderão ser minimizados e a funcionalidade nas diferentes áreas do desempenho ocupacional, ampliada.

\section{Referências}

ALBANESI-FILHO, F. M. A doença cardíaca, a insegurança e a disfunção sexual. Revista SOCERJ, Rio de Janeiro, v. 13, n. 3, p. 136-142, 2000.

ANDERSON, M. H.; CAMM, M. B. M. D. Legal and ethical aspects of driving and working in patients with an implantable cardioverter defibrillator. American Heart Journal, London, v. 127, n. 4, p. 1185-1193, 1994. Parte 2. ARTEAGA-FERNÁNDEZ, E. Cardiodesfibrilador implantável na prevenção de morte súbita na cardiomiopatia hipertrófica. 2011. 64 f. Tese (Livre-docência)-Faculdade de Medicina, Universidade de São Paulo, São Paulo, 2011.

BILGE, A. K. et al. Depression and anxiety status of patients with implantable cardioverter defibrillator and precipitating factors. Pacing and Clinical Electrophysiology, Mount Kisco, v. 29, n. 6, p. 619-626, 2006. PMID: 16784428 .

BARTHOLOMEW, T. et al. Working with elders who have cardiovascular conditions. In: PADILLA, R.; BEYERES-CONNON, S.; LOHMAN, H. Occupational therapy with elders: strategies for the cota. St Louis: Mosby, 2011. p. 72-79.

BRASIL, V. V.; CRUZ, D. A. L. M. Alteraçōes nos hábitos de vida relatadas por portadores de marcapasso definitivo. Revista Brasileira e Latinoamericana de 
Marcapasso e Arritmia, São Paulo, v. 13, n. 2, p. 97-113, 2000.

BRONZATTO, H. A.; SILVA, R. P.; STEIN, R. Morte súbita relacionada ao exercício. Revista Brasileira de Medicina Esporte, Niterói, v. 7, n. 5, p. 163-169, 2001.

CORDEIRO, J. J. R. Validação da lista de identificação de papéis ocupacionais em pacientes portadores de doença pulmonar obstrutiva crônica no Brasil. 2005. $111 \mathrm{f}$. Dissertação (Mestrado em Ciências da Saúde)-Escola Paulista de Medicina, Universidade Federal de São Paulo, São Paulo, 2005.

CORDEIRO, J. J. R. Cardiologia. In: CAVALCANTI, A.; GALVÃO, C. Terapia Ocupacional: fundamentação \& prática. Rio de Janeiro: Guanabara Koogan, 2007. p. 502-507.

CHAVES, G. F. S. et al. Escalas de avaliaçáo para Terapia Ocupacional no Brasil. Revista de Terapia Ocupacional, São Paulo, v. 21, n. 3, p. 240-246, 2010.

DANTAS, R. A. S.; AGUILAR, O. M.; BARBEIRA, C. B. S. Return to occupational and sexual activity after coronary artery bypass surgery. Revista Latino Americana de Enfermagem, Ribeirão Preto, v. 9, n. 4, p. 26-31, 2001. http://dx.doi.org/10.1590/S0104-11692001000400005

DURU, F. et al. How different from pacemaker patients are recipients of implantable cardioverter-defibrillators with respect to psychosocial adaptation, affective disorders, and quality of life? American Heart Journal, Saint Louis, v. 85, n. 4, p. 375-379, 2001.

EADS, A. S. et al. Supportive communication with implantable cardioverter defibrillator patients: seven principles to facilitate psychosocial adjustment. Journal of Cardiopulmonary Rehabilitation and Prevention, Philadelphia, v. 20, n. 2, p. 109-14, 2000. http://dx.doi. org/10.1097/00008483-200003000-00004

FERNÁNDEZ-BANIZI, P. et al. Interferencias electromagnéticas en pacientes con marcapasos y cardiodesfibriladores implantados. Revista médica del Uruguay, Montevideo, v. 20, n. 2, p. 150-160, 2004.

FONSECA, S. M. S. et al. Acompanhamento clínico de pacientes portadores de cardioversor-desfibrilador implantável. Arquivo Brasileiro de Cardiologia, Rio de Janeiro, v. 88, n. 1, p. 8-16, 2007.

HUNTLEY, N. Moléstias cardíacas e pulmonares. In: TROMBLY, C. A.; RADOMSKI, M. V. Terapia ocupacional para disfunções físicas. São Paulo: Editora Santos, 2005. p. 1071-1089.

KAMPHUIS, H. C. et al. Implantable cardioverter defibrillator recipients: quality of life in recipients with and without ICD shock delivery: a prospective study. Europace, London, v. 5, n. 4, p. 381-389, 2003. http:// dx.doi.org/10.1016/S1099-5129(03)00078-3

LEVY, R. F. et al. Teste cardiovascular aplicado ao consumo energético em atividades laborais. In: ENCONTRO NACIONAL DE ENGENHARIA DE PRODUÇÃO - ENEGEP, 1999, Rio de Janeiro. Anais.... Rio de Janeiro: ABEPRO, 1999. (Projeto do Posto e de Sistemas de Organização do Trabalho).

LIMA, C. E. B.; SILVA, R. T.; MARTINELLI FILHO, M. Indicaçôes de cardiodesfibrilador implantável e ressincronizador. São Paulo: Editorial MedicinaNet, 2009.

MACHADO, S. H.; PELÁ, N. T. R. Sexualidade e cardiopatia. Revista do Centro Universitário Barão de Mauá, Ribeirão Preto, v. 1, n. 1, p. 1-4, 2001.

RAJ, S. R.; SHELDON, R.S. Theimplantablecardioverterdefibrillator: does everybody need one?. Progress in Cardiovascular Diseases, New York, v. 44, n. 3, p. 169-94, 2001. PMid:11727276. http://dx.doi.org/10.1053/ pcad.2001.29146

SEARS JUNIOR, S. E.; CONTI, J. B. Understanding implantable cardioverter defibrillator shocks and storms: medical and psychosocial considerations for research and clinical care. Clinical Cardiology, New York, v. 26, n. 1, p. 107-111, 2003. PMID: 12685615.

SOLA, C. L.; B OS T W I K , J. M. Implantable cardioverter-defibrillators, induced anxiety, and quality of life. Mayo Clinic College of Medicine, Rochester, v. 80, n. 2, p. 232-237, 2005.

STEIN, R.; HOHMANN, C. B. Atividade sexual e coração. Arquivos Brasileiros de Cardiologia, São Paulo, v. 86 , n. 1, p. 61-67, 2006. http://dx.doi.org/10.1590/ S0066-782X2006000100010

TEIXEIRA, A. C. P.; FONSECA, A. R.; MAXIMO, I. M. N. S. Inventário SF36: avaliação da qualidade de vida dos alunos do Curso de Psicologia do Centro UNISAL - Universidade Estadual de Lorena (SP). Psic: revista da Vetor Editora, São Paulo, v. 3, n. 1, p. 16-27, 2002.

\section{Contribuição dos Autores}

Todas as autoras foram responsáveis pela concepção do texto, organização de fontes e análises, redação do texto e revisão.

\section{Notas}

${ }^{1}$ O Trabalho foi desenvolvido no Hospital Universitário Clementino Fraga Filho, RJ. Este material é parte de pesquisa com intervenção e foi apresentado como Trabalho de Conclusão de Curso pela Residência Multiprofissional e Integrada da UFRJ. Todos os procedimentos éticos necessários foram realizados. 